\title{
Chief Physician Defen Wang's Clinical Experience in Dialectical Treatment with Mediation Method of Perimenopausal Sweating
}

\author{
Mengyu Zhang1, Defen Wang2*, Tian Zhao' ${ }^{1}$ \\ ${ }^{1}$ Department of Integrated Traditional Chinese and Western Medicine, Shaanxi University of Chinese Medicine, Xianyang \\ 711301, Shaanxi Province, China \\ ${ }^{2}$ Department of Endocrinology, Xi'an Hospital of Traditional Chinese Medicine, Xi'an 710021, Shaanxi Province, China \\ *Corresponding author: Defen Wang, wangdefen69@sohu.com \\ Copyright: () 2022 Author(s). This is an open-access article distributed under the terms of the Creative Commons Attribution License (CC \\ BY 4.0), permitting distribution and reproduction in any medium, provided the original work is cited.

\begin{abstract}
The objective of this paper is to outline Chief Physician Defen Wang's clinical experience in perimenopausal sweating syndrome differentiation and treatment, to inherit Mr. Gao's academic concept of mediation technique, and to integrate multiple prescriptions under Mr. Gao's thinking. The deficiency is based on the deficiency of liver and kidney Yin, the weakness of spleen and stomach, and the standard is the imbalance of Ying and Wei, the imbalance of Qi, blood and body fluid, and the main method of treatment is nourishing liver and kidney, soothing liver and invigorating spleen, regulating Ying and Wei, as well as tonifying Qi and absorbing fluid.
\end{abstract}

Keywords: Sweating syndrome; Nourishing liver and kidney; Nourish the liver and invigorate the spleen; Perimenopausal period; Balancing method

Online publication: January 19, 2022

\section{Introduction}

Perimenopausal sweating syndrome is a frequent condition that occurs before and after perimenopausal period. Menstrual cycle problem, hot flashes and sweating, irritability, dizziness and palpitation, dizziness and tinnitus, waist and back discomfort, and weakness are all indications of a Yin and Yang imbalance before and after perimenopause. It is classified as "perimenopausal syndrome," "depression syndrome," and "hysteria" in traditional Chinese medicine. Sweating syndrome is crucial to treat since it accounts for a high proportion of all disorders and severely interferes with patients' everyday lives ${ }^{[1]}$. Hormone replacement therapy is currently the first line of treatment for women suffering from perimenopause symptoms in Western medicine, although it has a number of adverse effects and contraindications. Patients prefer traditional Chinese medicine in clinical settings. The author was fortunate enough to receive instruction from Chief Physician Defen Wang. Mr Gao's academic notion of balancing technique was passed down to the author, who employed medications based on clinical syndrome distinction. The lack of liver and kidney Yin, the weakening of the spleen and stomach as the foundation, the imbalance of Ying and Wei, and the imbalance of Qi, blood and bodily fluid as the standard, she believed the disease was complicated, asthenia in origin and asthenia in superficiality.

The author believes that perimenopausal sweating is caused by liver and kidney Yin deficiency, as well as spleen and stomach weakness, and that the treatment method is "nourishing liver and kidney, 
supplementing Qi and strengthening spleen, harmonising Ying and Wei, tonifying Qi and absorbing fluid." "Danzhi Xiaoyao Powder," "Danggui Liuhuang Decoction," "Chaihu Shugan Powder," and "Sijunzi Decoction" are commonly used in clinical practice to treat the condition, and they are highly successful.

\section{Tracing the origin of "harmonization method" theory}

In ancient times, the word “和” was written in the style of “盉” and “掄禾.” In “Shuowenjiezizhu”: “盉” is used for blending the wine. The tone is the same as “劔禾” and the one that blends the wine is called a “盉." The original meaning of “㖮” is regulating sound, and the original meaning of “盉” is "regulating five tastes," which gradually converged into “和” ${ }^{[2]}$. According to the report, "“"the nose is related to the lung Qi, therefore the nose can detect scent and odor if the lung is in balance," says Lingshu Maidu. The tongue is related to the heart Qi, therefore if the heart is in balance, the tongue can detect the five tastes. Because the liver and the eyes are connected by Qi, if the liver is in balance, the liver and the eyes can identify the five colours. The spleen Qi is related to the mouth, thus if the spleen is in balance, the spleen and the mouth may both know the five grains. The kidney Qi is related to the ears, therefore if the ear is in balance, the kidney and the ears can distinguish the five tones ${ }^{[3]}$." It is considered that Yin and Yang, internal organs, Qi, blood and semen should achieve a balance state of interoperability, and "imbalance" is the emergence of disease. "Both Yin and Yang in balance" is a good description of "balance" in "Neijing."

The words "disease" and "balance" appear frequently in Zhongjing's Treatise on Febrile Diseases: "Yingqi balances by self-sweating in patients. Those who have Yingqi balanced, however, do not have Weiqi bala, Patiefrequently sweat because Weiqi and Yingqi are imbalanced." It states that Yingqi runs on pulse to assist Weiqi, while Weiqi runs against pulse to strengthen the Yingqi and blood, and that if Weiqi is insufficient, it loses its strength, causing sweating and imbalance. To reconcile Ying and Wei, they utilise Guizhi Decoction ${ }^{[4]}$. In his medical enlightenment during the Qing Dynasty, Guopeng Cheng clearly stated the "eight techniques of medicine," which included sweat, vomit, purgation, harmony, warm, clear, elimination, and tonic. The concept of "balancing technique" was formally developed, and it was officially acknowledged as one of the major treatment methods of traditional Chinese medicine ${ }^{[5]}$.

Professor Shanglin Gao, who advocates the theory of the unity of heaven and man, is the author's mentor. He thinks that Yin and Yang are in balance, that Qi and blood are in balance, that blood essence is sufficient, that internal organs are in balance, and that no diseases exist. On the contrary, disease will arise if blood and oxygen do not work together. Mr. Gao develops the hypothesis that "if the human body is out of balance, all illnesses would emerge." He believes that the main principle of management is balance, and that all techniques are balanced. The first of the eight procedures is balancing. Mr. Gao argues that the Xiaochaihu Decoction described by Zhongjing in his "Treatise on Febrile Diseases" is "the Shaoyang pivot machine's medicine and the general prescription for reconciling the exterior and the interior" ("Shanghanlaisuji Hanfiyi"). It has been continually modified and developed, although its essential has remained unchanged. It has developed a set of prescriptions based on the compatibility of Bupleurum and Pinellia ternata, with functions such as soothing Yin and Yang, regulating viscera, balancing Qi and blood, resolving both the exterior and the interior, employing cold and heat, attacking and replenishing ${ }^{[6]}$.

\section{Etiology and pathogenesis of perimenopausal sweating}

"When the woman becomes 49 years old, the Qi and blood of Ren meridian began to be weak, Taichong meridian began to decline, and Tiangui dries up," according to "The Inner Canon of Huangdi." The liver can no longer be nourished by the kidney Yin, the Yin of the liver and kidney reduces, the kidney function slows down, the water fails to moisten kidney, it cannot restrict the liver Yang and clear orifices to make people dizzy, and weakening of kidney causes fire-syndrome, forcing the body fluid to leak, resulting the patient to sweat. "At the age of 50, liver Qi began to reduce, and liver lobes began to narrow," 
according to "Lingshu Tiannian." It emphasizes that women take the liver as the congenital factor and utilizes the liver blood through pregnancy and childbirth, then the liver loses its nourishment, and causes the liver Qi to be easy to be deficient, the liver governs the diffusion, the function is good, the liver is over worked and the liver blood is insufficient, the instability is prolonged and the heat is transitioned. The lung Qi is affected, the lung function convergence is insufficient, the Wei is not stable, the semen is lost, and the muscular surface is flooded all at the same time ${ }^{[7]}$." "Dongyuan said: In the southwest lies Kun soil," according to "The True Story of Medicine Sweat Syndrome." The spleen and stomach are located in the human body. The sweat of huma is like the rain of heaven and earth. If Yin's humidity is nourished, it produces fog and dew, as well as rain. It is different from the heart, according to the internal classic, while Dongyuan refers to the spleen and stomach. This is because the heart is in charge of fire and dominates heat, while the spleen and stomach are in charge of earth and moisture. Thus, moisture and heat fights for dominance and causes sweat." "Therefore, when you are full of food, sweat comes from your stomach," "Suwen, Jingmaibielun" added. Sweat emerges from your spleen when you shake your body and work hard. As a result, perimenopausal sweating is linked to the liver and kidney, spleen and stomach, as well as Ying and Wei. It may be described as yin deficiency of the liver and kidneys, spleen and stomach weakness, Ying and Wei imbalance, and Qi, blood, and bodily fluid imbalance.

\section{Chief Physician Defen Wang's experience in dialectical treatment}

"Yin deficit of the liver and kidney, weakening of the spleen and stomach, loss of Ying and Wei, imbalance of Qi, blood and bodily fluid" are the aetiology and pathophysiology of abnormal sweating in perimenopause. Professor Defen Wang mixed "Danzhi Xiaoyao Powder," "Danggui Liuhuang Decoction," "Chaihu Shugan powder," and other formulas with "Sijunzi Decoction" based on her expertise. Medicine used was: Paeonia suffruticosa, Fructus Gardeniae, Radix Angelicae Sinensis, Radix Paeoniae Alba, roasted Rhizoma Atractylodis Macrocephalae, Poria cocos, Rhizoma Anemarrhenae, wine Cornus, Radix Bupleuri, Schisandra chinensis, cortex lycii radicis, Radix Astragali seu Hedysari and Radix Glycyrrhizae. Danzhi Xiaoyao powder first appeared in the "Internal Medicine Abstract" of Ji Xue of Ming Dynasty. Xue included paeonia suffruticosa and fried capejasmine to the Song Dynasty's “Taiping Huimin Heju Prescription" to cure the syndrome of liver and spleen blood deficit based on the characteristics of liver disease ${ }^{[8]}$. The soup Danggui Liuhuang comes from Dongyuan Li's "Lanshi Mizang." The entire formula contains seven tastes, with the purifying hot medicine, relaxing Yin blood, and reducing night sweats being the most important. Therefore, it is referred to as "the holy medication for treating night sweats ${ }^{[9] " ~ b y ~}$ "Lashi Mizang." The primary prescription for strengthening the spleen and replenishing Qi is Sijunzi Decoction. Radix Ginseng, Rhizoma Atractylodis Macrocephalae, Poria cocos, and Radix Glycyrrhizae have four distinct textures that are warm but not dry, minty but not bitter, and in keeping with Mr. Gao's philosophy of "taking balance as the first."

Other concurrent syndromes in perimenopausal women include Yin deficiency, excessive heat, internal blockage of phlegm and dampness, heart and spleen deficiency, and other frequent syndrome types, all of which necessitate dialectical prescription selection to add or withdraw medication ${ }^{[10]}$. Paroxysmal hot sweat, agitation, and irritability are all symptoms of yin deficit and heat. The authors' mentor used Anemarrhena asphodeloides, Cortex Phellodendri, Lophatherum gracile, cortex lycii radicis and other drugs more clinically. Internal phlegm-moisture resistance causes heavy head, lack of sensation on face, bloated limbs, chest and diaphragm stuffiness, and poor mental concentration. Professor has a lot of experience with Rhizome Pinelliae Preparata, dried orangepeel, Semen Coicis, Cortex Magnoliae Officinalis, Fructus Amomi Villosi, Rhizoma Atractylodis, and other herbs. Palpitation, chest tightness, lack of smoothness, insomnia and dreams, mental fatigue, forgetfulness, apathetic expression, eating little, and loose stool are all signs of heart and spleen weakness. Radix Astragali seu Hedysari, fried Semen 
Ziziphi Spinosae, Radix Angelicae Sinensis, calcined oysters, and other ingredients can be added.

\section{Examples of medical records}

Ms. Li is a 49-year-old female patient. For the last two years, the main complaint has been full body night sweat and spontaneous sweat. The patient is now surrounded by heat, which promotes sweating, and gets worse after movement. She sweats at night, feels cold after sweating, has a weak physique, a sore back, a dry mouth, and the need to drink cold water. The patient has recently been irritable and impatient, with no dizziness, nausea, or vomiting. She is also upset and has chest tightness. She has a low appetite, frequent urine, dry stool, poor night rest, and difficulty falling asleep. Her menstrual cycle is not in order. Her pulse string is narrow, her tongue is light red with a thin white layer.

First diagnosis was, sweat syndrome - liver and kidney Yin were weak, spleen and stomach were weak and Ying and Wei were imbalance. Treatment was, nourishing liver and kidney, soothing liver and strengthening spleen, balancing Ying and Wei. Prescription selected was, adjustment of Danzhi Xiaoyao Powder, 15g medicinal paeonia suffruticosa, 15g Radix Angelicae Sinensis, 10g vinegar Radix Bupleuri, 15g Radix Paeoniae Alba, 15g fried Rhizoma Atractylodis Macrocephalae, 6g Radix Glycyrrhizae, 6g Fructus Gardeniae Praeparatus, 10g salt anemarrhenae rhizoma, 10g wine Cornus, $15 \mathrm{~g}$ Poria cocos, $12 \mathrm{~g}$ cortex lycii radicis, $20 \mathrm{~g}$ poria with hostwood, $15 \mathrm{~g}$ fried Semen Ziziphi Spinosae, 10g Rhizome Pinelliae Preparata, 20g salt Eucommia ulmoides, 6 doses, 1 dose/day, decocted in 400ml water, taken warm twice in the morning and evening. Patients should avoid consumption of raw, cold, spicy and stimulating products. However, they should exercise moderately, regulate their emotions, etc.

The second diagnosis was that the heat sweats were greatly reduced after taking the above medication. Her dry mouth has improved, as well as her irritation, and she is no longer experiencing headaches, chest tightness, or other problems. She can eat, sleeps through the night, and has two stools on a regular basis. If the patient continues to sweat profusely after moving, feels back discomfort, and is sleepy. Then, to the original prescription, we add 15g Radix Astragali seu Hedysari and 12g Schisandra chinensis, remove the cortex lycii radicis and Radix Paeoniae Alba, and continue to take 6 doses.

The patient's sweating was greatly reduced, her general fatigue was reduced, her waist and back discomfort went, she had mild abdominal cramping, food intake, night rest, and two stool modifications, according to the third diagnosis. From the original prescription, remove the salt Eucommia ulmoides, poria with hostwood, and add 10g Cortex Magnoliae Officinalis. Six dosages were given to the patient. The patient was told to keep a positive attitude and exercise. The patient's hot sweat did not return after 3 months of follow-up.

\section{Case evaluation}

Based on the patient's menstrual history and other relevant examinations, the patient was diagnosed with perimenopausal syndrome with hot sweat as the predominant symptom. Danzhi Xiaoyao Powder is chosen based on the patient's symptoms, signs, tongue coating, and pulse, as well as the patient's Tiangui being exhausted, the lack of Qi and blood, liver and kidney Yin weakening, liver failure, and spleen weakness. The patient feels hot all over, agitated, and has to urine frequently and dry stool. To remove heat, salt Rhizoma Anemarrhenae and cortex lycii radicis were added to the pyretolysis process. Revive the liver and kidneys with salt Eucommia ulmoides and wine Cornus. Pinelliae Preparata Rhizome dried the moisture and cleared the phlegm. During the second diagnosis, the symptoms were significantly improved, the sweating was significantly reduced, the cortex lycii radicis and Radix Paeoniae Alba were removed, and Radix Astragali seu Hedysari and Schisandra chinensis were added to help stop sweating. During the third diagnosis, the symptoms basically disappeared, but there was a slight abdominal distention, so Cortex Magnoliae Officinalis was added on the basis of the original prescription to eliminate stagnation of Qi. 


\section{Funding}

Shanglin Gao national famous traditional Chinese medicine inheritance studio construction project of the National Administration of Traditional Chinese Medicine (Department of Human Education, State Administration of Traditional Chinese Medicine [2011] No. 41); Key research and development projects of Shaanxi Province in 2020 (2020SF-276); Science and technology project of Xi'an Health Bureau (J201902018).

\section{Disclosure statement}

The author declares no conflict of interest.

\section{References}

[1] Huang Y, Shi H, Luo W, 2017, Analysis of Perimenopausal Sweat Syndrome. Lishizhen Medicine and Materia Medica Research. 28(11): 2710-2711.

[2] Gao P, Ju B, 2019, Discussion on "Harmonization Method" in Huangdi Neijing and Treatise on Febrile Diseases. Journal of Practical Traditional Chinese Internal Medicine, 33(05): 10-12.

[3] Zhang W, He X, 2005, "Harmony" of Philosophy and "Harmony" of Traditional Chinese Medicine. Knowledge of Ancient Medical Literature, (04): 4-7.

[4] Li W, Su T, Ke X, 2020, Analysis of Relevant Prescriptions and Syndromes of Sweat Syndrome in Treatise on Febrile Diseases. Xinjiang Journal of Traditional Chinese Medicine, 38(03): 56-57.

[5] Qin J, Chen L, 2014, Zhang Jingyue's Difference in "Harmonizatio Method" in Distinguishing himself from Small Bupleurum Decoction. Lishizhen Medicine and Materia Medica Research. 25(12): 29842985.

[6] Bai X, Pei R, 2013, Discussion on the Thought of Chief Physician Gao Shanglin and Law. Western Journal of Traditional Chinese Medicine, 26(06): 24-26.

[7] Li P, Yu B, 2019, Professor Yu Baili's Clinical Experience in the Treatment of Perimenopausal Abnormal Sweating by Tonifying the Spleen and Absorbing Fluid. Guangming Journal of Chinese Medicine, 34(09): 1333-1334.

[8] Jia L, Feng Z, 2017, Professor Feng Zhihai's Experience in Treating Perimenopausal Syndrome with Danzhi Xiaoyao Powder. Chinese Journal of Ethnomedicine and Ethnopharmacy. 26(06): 71-72.

[9] Li C, Wang S, Li Z, 2017, Professor Li Zhen's Experience in Treating Climacteric Sweat Syndrome with Danggui Liuhuang Decoction. Guangming Journal of Chinese Medicine, 32(08): 1096-1097.

[10] Hao C, Zhou Q, He Y, et al., 2018, Professor Zhou Qing's Clinical Experience in Treating Premenopausal and Postmenopausal Syndromes based on Syndrome Differentiation. Yunnan Journal of Traditional Chinese Medicine and Materia Medica, 39(04): 9-10. 